\title{
Morbidade em candidatos a emprego na região metropolitana de Salvador, Bahia, Brasil
}

\author{
Morbidity among job applicants in Greater \\ Metropolitan Salvador, Bahia, Brazil
}

Vilma S. Santana 1

Lilian C. Carvalho 1

Cristina P. Santos 1

Charles de Andrade 1

Gilma D'Oca ${ }^{1}$

\footnotetext{
1 Instituto de Saúde Coletiva, Universidade Federal da Bahia. Rua Padre Feijó 29, 4 o andar, Salvador, $B A$ 40110-170, Brasil. vilma@ufba.br
}

\begin{abstract}
This cross-sectional study evaluates the pre-employment health status of job applicants, based on prevalence estimates of abnormal laboratory test results, clinical diagnosis, and a physician-signed fit-for-work assessment, which is mandatory in Brazil. The study population was a random sample of 1,237 male workers selected from medical records (1988-1996) from an Occupational Medical Center located in Salvador, the capital of Bahia State, Brazil. The data were from a single pre-employment medical assessment for each individual. The most common diseases were intestinal parasites (51.7\%), anemia (12.8\%), and hypertension (12.7\%). Drivers showed a high prevalence of overweight (38.2\%) and hypertension (16.2\%). In addition to workrelated risks and diseases, occupational hygiene and safety programs need to address prevailing illnesses and risk factors, which in this population are typical of poor living conditions. Special attention should to be given to drivers, who are at increased risk of cardiovascular disease.
\end{abstract}

Key words Occupational Health; Morbidity; Prevalence; Workers; Employment

Resumo O objetivo deste estudo transversal é avaliar o perfil de morbidade de candidatos a emprego, através de estimativas de prevalência de alterações de exames laboratoriais, dados da anamnese clínica e do laudo médico ocupacional sobre a aptidão para o trabalho. A população do estudo se constitui de 1.237 trabalhadores do sexo masculino, selecionados aleatoriamente através de prontuários médicos de um Serviço de Medicina Ocupacional de Salvador-Bahia, no período entre 1988 e 1996. Apenas dados de exames admissionais foram utilizados. As enfermidades mais encontradas foram infecções parasitárias (51,7\%), anemias (12,8\%) e hipertensão arterial (12,7\%), enquanto que o sobrepeso atingiu 24,7\% da população do estudo. Motoristas apresentaram altas prevalências de sobrepeso (38,2\%) e hipertensão arterial (16,2\%). Programas de higiene e segurança no trabalho necessitam enfocar, além de riscos e doenças ocupacionais, as mais comuns enfermidades e riscos, que nesta população foram aqueles típicos das más condições de vida. Atenção especial para a prevenção de doenças cardiovasculares deve ser dirigida a motoristas.

Palavras-chave Saúde Ocupacional; Morbidade; Prevalência; Trabalhadores; Emprego 


\section{Introdução}

No Brasil, dados de morbidade de trabalhadores inexistem, exceto para alguns grupos ocupacionais específicos como metalúrgicos (Klein et al., 1986; Bertolami et al.,1993) ou motoristas de ônibus (Cordeiro, 1991). Avaliações clínicas admissionais, periódicas e demissionais constituem a base do monitoramento da saúde no trabalho, obrigatório para os trabalhadores do setor formal da economia (Brasil, 1978). Além da seleção de candidatos aptos para as funções oferecidas pelo mercado de trabalho, através da verificação da compatibilidade entre o nível de saúde física e mental do trabalhador, com a atividade a ser exercida, estes dados deveriam ser utilizados como instrumentos efetivos de vigilância à saúde dos trabalhadores. Entretanto, resultados dessas avaliações médicas são raramente objeto de análises epidemiológicas, que poderiam subsidiar a formulação de programas de intervenção para a melhoria das condições de trabalho e de saúde desses grupos populacionais.

Neste estudo, o perfil epidemiológico de saúde da população demandante por trabalho de diferentes setores da economia, é analisado com base nos exames admissionais através de prevalências de resultados de exames laboratoriais e complementares anormais, dados do estado vital, queixas referidas e diagnóstico clínico, com as suas características descritivas em relação à idade, sexo, nível de qualificação ocupacional e ramo da atividade à qual o candidato se habilita.

\section{Metodologia}

Este é um estudo transversal realizado com candidatos a emprego, examinados entre 1o de janeiro de 1988 e 31 de dezembro de 1996, em uma das unidades de saúde de um Serviço de Medicina Ocupacional da Região Metropolitana de Salvador. Esses indivíduos são encaminhados por indústrias e empresas de serviços e comércio de pequeno e médio porte, com algumas exceções nas indústrias de grande porte, como a petroquímica, localizadas, em geral, na própria Região Metropolitana de Salvador, e que contratam serviços médico ocupacionais e laboratoriais com essa instituição.

Os exames admissionais ocorrem antes da contratação para a ocupação de vagas emergentes, ou para a formalização do contrato de trabalhadores empregados temporariamente. Compreendem uma avaliação clínica semi-estruturada e uma bateria de exames laborato- riais padronizada que, além da conclusão diagnóstica, acompanham-se de um laudo ocupacional que classifica os sujeitos em apto, não apto e parcialmente apto para a função. A rotina de avaliação admissional se inicia com entrevistas para registro dos dados pessoais, realização dos exames laboratoriais e aprazamento da consulta médica e odontológica. Exames laboratoriais ou complementares, além dos obrigatórios, podem ser solicitados para esclarecimento de dúvidas diagnósticas, como a audiometria e o eletrocardiograma.

A população do estudo é uma amostra aleatória de 1.237 candidatos, de um total estimado de 61.000 , que apresentavam o registro de pelo menos um exame admissional realizado em data compreendida dentro do período do estudo. Devido às complexas diferenças de gênero relativas à morbidade, a análise desse estudo é restrita para os homens, enquanto que as mulheres estão sendo objeto de um outro trabalho. Para a coleta dos dados, utilizou-se um formulário pré-codificado que foi preenchido por nutricionistas e estudantes de medicina treinados. Da anamnese clínica anotou-se a pressão arterial, o peso e a altura, história de consumo de bebidas alcoólicas e de tabaco, a queixa principal, o diagnóstico clínico e o laudo ocupacional. Os exames laboratoriais padronizados foram o parasitológico de fezes, o hemograma completo e o sumário de urina.

Com essas informações, definiram-se as seguintes variáveis para o estudo: queixa clínica referida (queixa principal), anemia (hemoglobina $<13,5 \mathrm{mg} / \mathrm{dl}$, valor normal segundo Miller, 1991) alteração do exame sumário de urina (anormal: piócitos acima de 10 por campo ou qualquer outro dado alterado), parasitoses intestinais, sobrepeso baseado no Índice de Massa Corporal, que corresponde ao peso em quilogramas dividido pelo quadrado da altura em metros, acima do ideal $\geq 25,0$ (World Health Organization Consultation on Obesity, 1998), consumo de bebidas alcoólicas, consumo de tabaco e hipertensão arterial (pressão arterial sistólica $\geq 140 \mathrm{mmHg}$ e/ou pressão arterial diastólica $\geq 90 \mathrm{mmHg}$, valor de referência baseado no NIH et al., 1997), diagnóstico positivo e/ou tratamento específico atual registrado. As variáveis sócio-demográficas e ocupacionais foram: idade, classificada em grupos de 14 a 25, 26 a 32 e acima de 32 anos, tipo da ocupação e ramo da empresa para a qual o trabalhador se candidatava para emprego. Nível sócio-econômico foi definido de acordo com o nível de qualificação das ocupações (Singer, 1976): 1) Baixo - agentes de limpeza, serventes e ajudantes em geral; 2) Médio - operadores, auxiliares de 
produção, trabalhadores de manutenção industrial; 3) Alto - ocupações de nível educacional superior, gerência ou supervisão. Os ramos de atividade das empresas foram agrupados em setores de: 1) transportes; 2) indústrias de transformação; 3) construção civil; e 4) outros ramos não classificados nas categorias anteriores. As diversas ocupações foram agrupadas de acordo com a Standard Occupational Classification Manual (U. S. Department of Commerce, 1980) em: motoristas; cobradores; administração e ensino; operadores de máquinas e trabalhadores de produção; trabalhadores de mecânica e manutenção; limpeza e trabalhadores de construção.

Estimaram-se prevalências das variáveis relativas ao estado de saúde, de acordo com as variáveis descritoras, analisando-se as diferenças com o Teste do Qui-quadrado (Stockes et al., 1995). Devido à natureza exploratória desse estudo, destinado a orientar a programação de serviços de saúde, não se procedeu ajuste por idade ou outras variáveis.

\section{Resultados}

Dos 1.237 homens que compõem a população do estudo, a idade variou de 14 a 76 anos, com uma média de 30 anos, sendo a maioria $(48,9 \%)$ de nível sócio-econômico baixo (Tabela 1). A maior parte das empresas é do setor de transportes $(37,7 \%)$, seguida pelas indústrias de transformação $(36,8 \%)$, que juntas correspondem a mais de dois terços dos indivíduos estudados. Ainda na Tabela 1, pode-se observar que a ocupação mais comum é a de operadores de produção $(22,3 \%)$, seguida por motoristas $(16,1 \%)$, funções de administração/ensino (14,1\%), técnicos em mecânica/manutenção $(11,7 \%)$, cobradores (10,3\%), funções de limpeza $(6,9 \%)$ e trabalhadores da construção civil $(6,1 \%)$.

A prevalência de anemia segundo a faixa etária (Tabela 2) foi estimada em $12,8 \%$, de igual valor àquela estimada para alterações do exame sumário de urina (12, 8\%). As infecções por parasitos intestinais foram comuns, atingindo $51,7 \%$ da população do estudo. Destas infecções, as mais freqüentes foram por helmintos $(43,5 \%)$, em especial pelo Ascaris lumbricoides $(29,1 \%)$ e o Trichiurus trichiuri $(15,4 \%)$. Em geral, houve uma associação inversa da prevalência de parasitoses intestinais com a idade, embora tenha sido estatisticamente significante apenas para o conjunto dos parasitos e dos helmintos, para Giardia lamblia e A. lumbricoides $(\mathrm{p}<0,01)$, e para outras parasitoses $(\mathrm{p}<0,05)$. Verificou-se que $24,7 \%$ da população

\begin{tabular}{|c|c|c|}
\hline Dados sócio-demográficos dos car & a emprego. Salva & a, Brasil. \\
\hline Variáveis & $n(n=1.237)^{\star}$ & $\%$ \\
\hline \multicolumn{3}{|l|}{ Idade } \\
\hline 14 a 25 anos & 430 & 35,5 \\
\hline 26 a 32 anos & 370 & 30,5 \\
\hline$>32$ anos & 412 & 34,0 \\
\hline \multicolumn{3}{|l|}{ Nível sócio-econômico } \\
\hline Baixo & 595 & 48,9 \\
\hline Médio & 564 & 46,3 \\
\hline Mais/médio & 59 & 4,8 \\
\hline \multicolumn{3}{|l|}{ Setor } \\
\hline Transporte & 466 & 37,7 \\
\hline Indústria da transformação & 456 & 36,8 \\
\hline Construção civil & 127 & 10,3 \\
\hline Outros & 188 & 15,2 \\
\hline \multicolumn{3}{|l|}{ Ocupação } \\
\hline Operadores/produção & 276 & 22,3 \\
\hline Motoristas & 199 & 16,1 \\
\hline Administração/ensino & 174 & 14,1 \\
\hline Mecânica/manutenção & 145 & 11,7 \\
\hline Cobradores & 128 & 10,3 \\
\hline Limpeza & 85 & 6,9 \\
\hline Trabalhadores de construção & 76 & 6,1 \\
\hline Outros & 154 & 12,5 \\
\hline
\end{tabular}

$\mathrm{n}=$ Número de indivíduos

* Subtotais diferem para as variáveis idade e nível devido a indivíduos com informações perdidas (respectivamente 25 e 19 indivíduos).

do estudo apresentava sobrepeso (Índice de Massa Corporal acima de 25), e que esta prevalência aumentava com a idade, variando de $10,4 \%$ no grupo de 14 a 25 anos, para $26,2 \%$ (26 a 32 anos) e até $38,0 \%$ nos homens acima de 32 anos de idade $(\mathrm{p}<0,01)$. Além disso, $54 \%$ dos indivíduos referiram consumo de bebidas alcoólicas, sobretudo os do grupo entre 26 e 32 anos $(61,6 \%)$, enquanto que o tabagismo foi referido por $21,8 \%$ da população, aumentando com a idade $(p<0,01)$. Apesar de não se ter sido possível identificar os normotensos que ao exame representavam casos tratados, observou-se que $12,7 \%$ dos trabalhadores tiveram diagnóstico de hipertensão arterial, prevalência que também se eleva com a idade $(\mathrm{p}<0,01)$.

Na Tabela 3, pode-se observar que a maioria dos indicadores de morbidade apresentam tendência inversa com o nível sócio-econômico, embora apenas as parasitoses e helmintíases em geral e a infecção por A. lumbricoides apresentem diferenças estatisticamente signi- 
Prevalências de achados clínicos em candidatos a emprego, de acordo com a faixa etária. Salvador, Bahia, Brasil, 1988-1996.

\begin{tabular}{|c|c|c|c|c|c|c|c|c|}
\hline \multirow[t]{3}{*}{ Variáveis } & \multicolumn{6}{|c|}{ Faixa etária (anos) } & \multicolumn{2}{|c|}{ Total } \\
\hline & \multicolumn{2}{|c|}{$14-25$} & \multicolumn{2}{|c|}{$26-32$} & \multicolumn{2}{|c|}{$>32$} & & \\
\hline & $\mathrm{n}^{1}=430$ & $\%(35,5)$ & $\mathrm{n}=370$ & $\%(30,5)$ & $\mathrm{n}=412$ & $\%(34,0)$ & $\mathrm{n}=1.2122$ & $\%$ \\
\hline Anemia & 50 & 11,6 & 54 & 14,6 & 51 & 12,4 & 155 & 12,8 \\
\hline Sumário de urina anormal ${ }^{3}$ & 44 & 10,2 & 50 & 13,5 & 61 & 14,8 & 155 & 12,8 \\
\hline Todas as parasitoses ${ }^{\star \star}$ & 258 & 60,0 & 187 & 50,5 & 182 & 44,2 & 627 & 51,7 \\
\hline E. histolitica & 6 & 1,4 & 6 & 1,6 & 4 & 1,0 & 16 & 1,3 \\
\hline E. coli & 63 & 14,7 & 45 & 12,2 & 51 & 12,4 & 159 & 13,1 \\
\hline G. lamblia** & 22 & 5,1 & 6 & 1,6 & 9 & 2,2 & 37 & 3,1 \\
\hline Todos os helmintos** & 211 & 49,1 & 158 & 42,7 & 158 & 38,4 & 527 & 43,5 \\
\hline A. lumbricoides ${ }^{\star \star}$ & 150 & 34,9 & 98 & 26,5 & 105 & 25,5 & 353 & 29,1 \\
\hline T. trichiuri & 74 & 17,2 & 61 & 16,5 & 52 & 12,6 & 187 & 15,4 \\
\hline A. duodenalis & 30 & 7,0 & 18 & 4,9 & 22 & 5,3 & 70 & 5,8 \\
\hline S. stercoralis & 9 & 2,1 & 6 & 1,6 & 3 & 0,7 & 18 & 1,5 \\
\hline S. mansoni & 30 & 7,0 & 23 & 6,2 & 20 & 4,9 & 73 & 6,0 \\
\hline Outras parasitoses* & 25 & 5,8 & 15 & 4,1 & 8 & 1,9 & 48 & 4,0 \\
\hline Sobrepeso $4, \star \star$ & 19 & 10,4 & 37 & 26,2 & 68 & 38,0 & 124 & 24,7 \\
\hline Consumo de álcool** & 206 & 47,9 & 228 & 61,6 & 220 & 53,4 & 654 & 54,0 \\
\hline Tabagismo** & 51 & 11,9 & 99 & 26,8 & 114 & 27,7 & 264 & 21,8 \\
\hline Hipertensão arterial| ${ }^{5, \star \star}$ & 21 & 5,1 & 40 & 11,1 & 87 & 22,1 & 148 & 12,7 \\
\hline
\end{tabular}

1n = número de indivíduos; 2 O total difere devido a dados perdidos de 25 pessoas para a idade;

3 Sumário de urina anormal - pelo menos um dos resultados anormal;

4 Sobrepeso $=$ Índice de Massa Corporal $\geq 25$; subtotal difere devido a dados perdidos

de 727 indivíduos para esta variável; 5 Havia 49 indivíduos com dados perdidos para hipertensão arterial.

${ }^{\star} p<0,01 ;{ }^{* \star} p<0,05$

ficantes ( $\mathrm{p}<0,01)$, assim como outras parasitoses $(\mathrm{p}<0,05)$. Ao contrário, uma associação positiva com o nível sócio-econômico foi encontrada para o sobrepeso e a hipertensão $(\mathrm{p}<0,01)$ e também para o consumo de álcool e tabagismo embora não estatisticamente significante. A distribuição da morbidade de acordo com o setor (Tabela 4), todavia, não mostra variações relevantes, exceção para o T. trichiuri de ocorrência mais alta entre os trabalhadores do setor de transportes $(18,5 \%)$ e indústria de transformação (16,0 \%) do que os demais setores $(\mathrm{p}<0,05)$.

Os dados da Tabela 5 revelam diferenciais de morbidade em relação à ocupação dignas de nota, a exemplo da alta prevalência de sobrepeso $(38,2 \%)$ e hipertensão arterial $(16,2 \%)$ entre motoristas. Notam-se também as baixas freqüências de achados clínicos em trabalhadores de função administrativa e ensino quando comparados às demais ocupações, à exceção da hipertensão arterial, que teve prevalência de $16,6 \%$ nesta categoria.

\section{Discussão}

Com base nos dados desse estudo, pode-se afirmar que a população masculina que se candidata a emprego em Salvador, e que compõe a demanda desta unidade de medicina ocupacional, que cobre cerca de 6.000 empresas, apresenta altas prevalências de síndromes carenciais como a anemia e doenças transmissíveis como as parasitoses intestinais, que refletem o pobre acesso ao saneamento básico, água potável e precários hábitos de higiene. Dados sobre a ocorrência de anemia entre adultos em idade produtiva são raros, mas em um inquérito conduzido no México (González et al., 1998) foi observada uma prevalência de anemia de $11,1 \%$, próxima ao valor estimado neste estudo. As prevalências das parasitoses intestinais deste estudo são semelhantes aos de um outro estudo brasileiro (Ferreira et al., 1994), onde se observou uma prevalência de $45,7 \%$ de parasitoses, sendo também a ascaridíase a mais comum (23,8\%), seguindo-se pelo T. trichiuri que afetou $17,2 \%$ dos indivíduos. Semelhantes acha- 
Prevalências de achados clínicos entre candidatos a emprego, de acordo com o nível sócio-econômico. Salvador, Bahia, Brasil, 1988-1996.

\begin{tabular}{|c|c|c|c|c|c|c|c|c|}
\hline \multirow[t]{3}{*}{ Variáveis } & \multicolumn{6}{|c|}{ Nível sócio-econômico } & \multicolumn{2}{|c|}{ Total } \\
\hline & \multicolumn{2}{|c|}{ Baixo } & \multicolumn{2}{|c|}{ Médio } & \multicolumn{2}{|c|}{ Mais/médio } & & \\
\hline & $\mathrm{n}^{1}=595$ & $\%(48,9)$ & $n=564$ & $\%(46,3)$ & $n=59$ & $\%(4,8)$ & $\mathrm{n}=1.2182$ & $\%$ \\
\hline Anemia & 87 & 14,6 & 64 & 11,4 & 5 & 8,5 & 156 & 12,8 \\
\hline Sumário de urina anormal ${ }^{3}$ & 67 & 11,3 & 85 & 15,1 & 4 & 6,8 & 156 & 12,8 \\
\hline Todas as parasitoses* & 347 & 58,3 & 264 & 46,8 & 20 & 33,9 & 631 & 51,8 \\
\hline E. histolitica & 10 & 1,7 & 6 & 1,1 & - & - & 16 & 1,3 \\
\hline E. coli & 85 & 14,3 & 69 & 12,2 & 5 & 8,5 & 159 & 13,1 \\
\hline G. lamblia & 18 & 3,0 & 19 & 3,4 & - & - & 37 & 3,0 \\
\hline Todos os helmintos* & 296 & 49,8 & 218 & 38,7 & 18 & 30,5 & 532 & 43,7 \\
\hline A. lumbricoides* & 206 & 34,6 & 139 & 24,7 & 10 & 17,0 & 355 & 29,2 \\
\hline T. trichiuri & 102 & 17,1 & 80 & 14,2 & 7 & 11,9 & 189 & 15,5 \\
\hline A. duodenalis & 37 & 6,2 & 32 & 5,7 & - & - & 69 & 5,7 \\
\hline S. stercoralis & 9 & 1,5 & 7 & 1,2 & 1 & 1,7 & 17 & 1,4 \\
\hline S. mansoni & 42 & 7,1 & 29 & 5,1 & 3 & 5,1 & 74 & 6,1 \\
\hline Outras parasitoses** & 33 & 5,6 & 13 & 2,3 & 2 & 3,4 & 48 & 3,9 \\
\hline Sobrepeso $4, \star \star$ & 46 & 18,5 & 64 & 29,4 & 10 & 30,3 & 120 & 24,0 \\
\hline Consumo de álcool & 310 & 52,1 & 314 & 55,7 & 34 & 57,6 & 658 & 54,0 \\
\hline Tabagismo & 119 & 20,0 & 136 & 24,1 & 11 & 18,6 & 266 & 21,8 \\
\hline Hipertensão arterial ${ }^{5, \star *}$ & 59 & 10,2 & 82 & 15,3 & 10 & 17,9 & 151 & 12,9 \\
\hline
\end{tabular}

$1 \mathrm{n}=$ número de indivíduos; 2 O total difere devido a dados perdidos para o nível sócio-econômico de 19 pessoas;

3 Sumário de urina anormal - pelo menos um dos resultados anormal;

4 Sobrepeso $=$ Índice de Massa Corporal $\geq 25$; subtotal difere devido a dados perdidos

de 727 indivíduos para esta variável; 5 Havia 49 indíviduos com dados perdidos para hipertensão arterial.

${ }^{\star} p<0,01 ;{ }^{* \star} p<0,05$.

dos a esse estudo são ainda a de tendência inversa com a idade e nível sócio-econômico. Infelizmente não se dispõe de dados que poderiam indicar o nível de gravidade dessas infecções parasitárias, ou sintomatologia clínica associada, que permitissem uma mais adequada identificação do seu impacto em saúde pública. Todavia, isto revela que mesmo trabalhadores do setor formal, que puderam barganhar um contrato de trabalho, vivem em condições de vida precárias. Além desse fato, é possível supor que esta situação de saúde basal pode deteminar maior susceptibilidade a exposições ocupacionais, a exemplo das descrições de que carcinógenos, existentes em alguns ambientes de trabalho industrial, apresentam sinergismo com estados crônicos de deficiência de nutrientes, como vitaminas, sais minerais ou a anemia (Pearce et al., 1996). Além disso, deficiências nutricionais como a anemia são conhecidas por comprometerem o estado de alerta e disposição física geral, contribuindo para uma redução da produtividade, maior predisposição a doenças ocupacionais e um possível au- mento de acidentes de trabalho (Maturu \& ILO, 1979; Gomes, 1982) como o verificado entre trabalhadores da construção civil no estudo de Melhado et al. (1983).

Simultaneamente aos problemas de saúde típicos da pobreza, os achados de alta prevalência de fatores de risco para doenças crônicas não transmissíveis como o sobrepeso, o consumo de álcool, tabaco e a hipertensão arterial foram semelhantes aos dados encontrados em estudos nacionais (Lessa, 1998). Vale notar que em geral estes fatores de risco aumentavam com o nível sócio-econômico, a exceção do tabagismo, mais comum entre os mais pobres, indicando que categorias ocupacionais mais qualificadas também devem ser alvo de programas de prevenção para as doenças crônicas não transmissíveis.

Não houve variações significativas do estado de saúde em relação aos ramos da indústria para as variáveis analisadas nesse estudo, mas sim para as ocupações. Isto possivelmente resulta da grande heterogeneidade social dos setores que agrupam distintos segmentos da hie- 
Prevalências de achados clínicos em candidatos a emprego, de acordo com o setor. Salvador, Bahia, Brasil, $1988-1996$.

\begin{tabular}{|c|c|c|c|c|c|c|c|c|c|c|}
\hline \multirow[t]{3}{*}{ Variáveis } & \multicolumn{8}{|c|}{ Setor } & \multicolumn{2}{|c|}{ Total } \\
\hline & \multicolumn{2}{|c|}{ Transporte } & \multicolumn{2}{|c|}{ Transformação } & \multicolumn{2}{|c|}{ Construção civil } & \multicolumn{2}{|c|}{ Outros } & \multirow[b]{2}{*}{$\mathrm{n}=1.237$} & \multirow[b]{2}{*}{$\%$} \\
\hline & $\mathrm{n}^{1}=466$ & $\%(37,7)$ & $n=456$ & $\%(36,9)$ & $n=127$ & $\%(10,3)$ & $\mathrm{n}=188$ & $\%(15,2)$ & & \\
\hline Anemia & 66 & 14,2 & 61 & 13,4 & 9 & 7,1 & 23 & 12,2 & 159 & 12,9 \\
\hline Sumário de urina anormal2 & 53 & 11,4 & 68 & 14,9 & 17 & 13,4 & 21 & 11,2 & 159 & 12,9 \\
\hline Parasitoses intestinais & 259 & 55,6 & 232 & 50,9 & 65 & 51,2 & 85 & 45,2 & 641 & 51,8 \\
\hline E. histolitica & 7 & 1,5 & 4 & 0,9 & 2 & 1,6 & 3 & 1,6 & 16 & 1,3 \\
\hline E. coli & 72 & 15,5 & 51 & 11,2 & 18 & 14,2 & 22 & 11,7 & 163 & 13,2 \\
\hline G. lamblia & 19 & 4,1 & 10 & 2,2 & 3 & 2,4 & 5 & 2,7 & 37 & 3,0 \\
\hline Todos os helmintos & 213 & 45,7 & 202 & 44,3 & 56 & 44,1 & 70 & 37,2 & 541 & 43,7 \\
\hline A. lumbricoides & 136 & 29,2 & 134 & 29,4 & 39 & 30,7 & 53 & 28,2 & 362 & 29,3 \\
\hline T. trichiuri* & 86 & 18,5 & 73 & 16,0 & 11 & 8,7 & 24 & 12,8 & 194 & 15,7 \\
\hline A. duodenales & 25 & 5,4 & 28 & 6,1 & 9 & 7,1 & 8 & 4,3 & 70 & 5,7 \\
\hline S. stercoralis & 7 & 1,5 & 7 & 1,5 & 2 & 1,6 & 2 & 1,1 & 18 & 1,5 \\
\hline S. mansoni & 27 & 5,8 & 30 & 6,6 & 11 & 8,7 & 7 & 3,7 & 75 & 6,1 \\
\hline Outras parasitoses & 18 & 3,9 & 16 & 3,5 & 5 & 3,9 & 9 & 4,8 & 48 & 3,9 \\
\hline Sobrepeso 3 & 48 & 25,7 & 43 & 25,2 & 19 & 27,9 & 15 & 17,9 & 125 & 24,5 \\
\hline Consumo de álcool & 248 & 53,2 & 257 & 56,4 & 60 & 47,2 & 102 & 54,3 & 667 & 53,9 \\
\hline Tabagismo & 114 & 24,5 & 97 & 21,3 & 24 & 18,9 & 36 & 19,2 & 271 & 21,9 \\
\hline Hipertensão arterial4 & 47 & 10,4 & 63 & 14,4 & 18 & 15,3 & 25 & 14,0 & 153 & 12,9 \\
\hline
\end{tabular}

1n = número de indivíduos; 2 Sumário de urina anormal - pelo menos um dos resultados anormal;

3 Sobrepeso $=$ Índice de Massa Corporal $\geq 25$; subtotal difere devido a dados perdidos

de 727 indivíduos para esta variável; 4 Havia 49 indivíduos com dados perdidos para hipertensão arterial.

${ }^{\star} p<0,05 ;{ }^{\star *} p<0,01$.

rarquia ocupacional e também de nível de complexidade das firmas. Os grupos ocupacionais, no entanto, podem revelar padrões mais homogêneos, como por exemplo os motoristas que incluíam apenas os condutores de ônibus, tratores e caminhões que apresentaram uma alta prevalência de sobrepeso em relação à população do estudo, bem como de hipertensão arterial, semelhante ao encontrado no estudo de Ragland et al. (1987) sobre a prevalência de hipertensão em motoristas de ônibus. Este resultado de hipertensão e outros fatores de risco para doenças cardiovasculares em motoristas, têm sido alvo de pesquisas para busca de fatores causais como o estudo realizado por Belki'c et al. (1994) com motoristas profissionais. No estudo de Cordeiro (1991), a hipertensão arterial foi atribuída ao alto nível de estresse e sedentarismo próprios dessa ocupação. A importância destes dados reside no fato de que existem trabalhos que identificam associação entre doenças cardiovasculares e acidentes de trânsito em motoristas (Jovanovi'c et al., 1998, 1999) e maior severidade dos acidentes entre motoristas de ônibus hipertensos do que entre os saudáveis (Laberge-Nadeau et al., 1996), devendo-se assim considerar relevante o achado de fatores de risco para doenças crônicas não transmissíveis em exames admissionais de candidatos para esse cargo.

O quadro de morbidade encontrado é particularmente notável por se tratar de dados de exames admissionais, que podem refletir uma auto-seleção negativa, ou seja, enfermos conscientes do seu estado clínico podem evitar a realização desses exames, ou realizar tratamentos prévios como "preparo" para este tipo de avaliação médica. Apesar desses possíveis vieses de seleção que poderiam ter reduzido as prevalências das alterações da saúde, observou-se um perfil de morbidade que expressa a precariedade das condições de vida dos trabalhadores.

A utilização de dados secundários provenientes de um serviço de saúde ocupacional que realiza exames admissionais para um grande número de empresas do Estado, permitiu a obtenção de informação a baixo custo e curto prazo. Distintamente de estudos clínicos realizados com demanda atendida em serviços ambulatoriais especializados, que não possibili- 
Prevalências de achados clínicos em candidatos a emprego, de acordo com grupos ocupacionais. Salvador, Bahia, Brasil.

\begin{tabular}{|c|c|c|c|c|c|c|c|c|c|c|c|c|c|c|c|c|c|c|}
\hline \multirow[t]{3}{*}{ Variáveis } & \multicolumn{16}{|c|}{ Grupos ocupacionais } & \multirow{2}{*}{\multicolumn{2}{|c|}{ Total }} \\
\hline & \multicolumn{2}{|c|}{$\begin{array}{l}\text { Operadores } \\
\text { de produção }\end{array}$} & \multicolumn{2}{|c|}{ Motoristas } & \multicolumn{2}{|c|}{$\begin{array}{l}\text { Administração } \\
\text { e ensino }\end{array}$} & \multicolumn{2}{|c|}{$\begin{array}{l}\text { Mecânica e } \\
\text { manutenção }\end{array}$} & \multicolumn{2}{|c|}{ Cobradores } & \multicolumn{2}{|c|}{ Limpeza } & \multicolumn{2}{|c|}{$\begin{array}{l}\text { Trabalhadores } \\
\text { de construção }\end{array}$} & \multicolumn{2}{|c|}{ Outros } & & \\
\hline & $\begin{array}{l}\mathrm{n}^{1}= \\
209\end{array}$ & $\begin{array}{c}\% \\
(16,9)\end{array}$ & $\begin{array}{l}n= \\
199\end{array}$ & $\begin{array}{c}\% \\
(16,1)\end{array}$ & $\begin{array}{l}n= \\
174\end{array}$ & $\begin{array}{c}\% \\
(14,1)\end{array}$ & $\begin{array}{l}n= \\
145\end{array}$ & $\begin{array}{c}\% \\
(11,7)\end{array}$ & $\begin{array}{l}n= \\
128\end{array}$ & $\begin{array}{c}\% \\
(10,3)\end{array}$ & $\begin{array}{l}n= \\
85\end{array}$ & $\begin{array}{c}\% \\
(6,9)\end{array}$ & $\begin{array}{l}n= \\
29\end{array}$ & $\begin{array}{c}\% \\
(2,3)\end{array}$ & $\begin{array}{l}n= \\
268\end{array}$ & $\begin{array}{c}\% \\
(21,7)\end{array}$ & $\begin{array}{l}\mathrm{n}= \\
1.237\end{array}$ & $7^{\%}$ \\
\hline Anemia & 47 & 17,0 & 23 & 11,6 & 16 & 9,2 & 19 & 13,1 & 21 & 16,4 & 11 & 12,9 & 4 & 5,3 & 18 & 11,7 & 159 & 12,9 \\
\hline $\begin{array}{l}\text { Sumário de } \\
\text { urina anormal }{ }^{2}\end{array}$ & 35 & 12,7 & 28 & 14,1 & 19 & 10,9 & 22 & 15,2 & 13 & 10,2 & 13 & 15,9 & 10 & 13,2 & 19 & 12,3 & 159 & 12,9 \\
\hline Todas as parasitoses ${ }^{\star \star}$ & 49 & 54,0 & 98 & 49,3 & 66 & 37,9 & 83 & 57,2 & 74 & 57,8 & 53 & 62,4 & 34 & 54,6 & 84 & 54,6 & 641 & 51,8 \\
\hline E. histolitica & 1 & 0,4 & 1 & 0,5 & 3 & 1,7 & 2 & 1,4 & 2 & 1,6 & 2 & 2,4 & 1 & 1,3 & 4 & 2,6 & 16 & 1,3 \\
\hline E. coli & 8 & 10,1 & 28 & 14,1 & 19 & 10,9 & 19 & 13,1 & 20 & 15,6 & 13 & 15,3 & 10 & 13,2 & 26 & 16,9 & 163 & 13,2 \\
\hline G. lamblia & 7 & 2,5 & 6 & 3,0 & 5 & 2,9 & 6 & 4,1 & 6 & 4,7 & 2 & 2,4 & 2 & 2,6 & 3 & 2,0 & 37 & 3,0 \\
\hline Todos os helmintos** & 131 & 47,5 & 76 & 38,2 & 55 & 31,6 & 74 & 51,0 & 60 & 46,9 & 49 & 57,7 & 27 & 35,5 & 69 & 44,8 & 541 & 43,7 \\
\hline A. lumbricoides ${ }^{\star \star}$ & 88 & 31,9 & 8 & 24,1 & 32 & 18,4 & 50 & 34,5 & 40 & 31,3 & 34 & 40,0 & 21 & 27,6 & 49 & 31,8 & 362 & 29,3 \\
\hline T. trichiuri & 40 & 14,5 & 33 & 16,6 & 21 & 12,1 & 24 & 16,6 & 22 & 17,2 & 18 & 21,2 & 8 & 10,5 & 28 & 18,2 & 194 & 15,7 \\
\hline A. duodenales & 19 & 6,9 & 12 & 6,0 & 6 & 3,5 & 12 & 8,3 & 5 & 3,9 & 9 & 10,6 & 2 & 2,6 & 5 & 3,3 & 70 & 5,7 \\
\hline S. stercoralis & 4 & 1,5 & 2 & 1,0 & 3 & 1,7 & 3 & 2,1 & 2 & 1,6 & 2 & 2,4 & - & - & 2 & 1,3 & 18 & 1,5 \\
\hline S. mansoni & 13 & 4,7 & 9 & 4,5 & 8 & 4,6 & 14 & 9,7 & 6 & 4,7 & 9 & 10,6 & 5 & 6,6 & 11 & 7,1 & 75 & 6,1 \\
\hline Outras parasitoses & 11 & 4,0 & 7 & 3,5 & 4 & 2,3 & 4 & 2,8 & 4 & 3,1 & 7 & 8,2 & 3 & 4,0 & 8 & 5,2 & 48 & 3,9 \\
\hline Sobrepeso ${ }^{3, *}$ & 19 & 17,8 & 26 & 38,2 & 24 & 32,0 & 16 & 23,9 & 6 & 11,1 & 8 & 20,5 & 8 & 23,5 & 18 & 27,3 & 125 & 24,5 \\
\hline Consumo de álcool & 154 & 55,8 & 116 & 58,3 & 102 & 58,6 & 82 & 56,6 & 57 & 44,5 & 44 & 51,8 & 38 & 50,0 & 74 & 48,1 & 667 & 53,9 \\
\hline Tabagismo & 55 & 19,9 & 54 & 27,1 & 37 & 21,3 & 34 & 23,5 & 21 & 16,4 & 18 & 21,2 & 20 & 26,3 & 32 & 20,8 & 271 & 21,9 \\
\hline Hipertensão arterial ${ }^{4}$ & 37 & 13,9 & 31 & 16,2 & 27 & 16,6 & 13 & 9,4 & 9 & 7,2 & 8 & 10,0 & 13 & 18,1 & 15 & 9,9 & 153 & 12,9 \\
\hline
\end{tabular}

$1 \mathrm{n}=$ número de indivíduos; 2 Sumário de urina alterado - pelo menos um resultado anormal;

3 Sobrepeso = Índice de Massa Corporal $\geq 25$; subtotal difere devido a dados perdidos

de 727 indivíduos para esta variável; 4 Havia 49 indíviduos com dados perdidos para hipertensão arterial.

${ }^{*} p<0,05 ;{ }^{* *} p<0,01$.

tam a estimativa de indicadores epidemiológicos por falta de denominador adequado, dados de triagens populacionais como estes, permitem a caracterização do estado vital e a morbidade de patologias comuns. Esses dados poderão subsidiar a elaboração de políticas de atenção e promoção à saúde do trabalhador desempregado, ou que se encontra em processo de mudança de emprego, permitindo medidas mais apropriadas, e preparando-o para a participação na força de trabalho ativa.

As principais limitações desta pesquisa residem na natureza secundária dos seus dados, especialmente por serem provenientes de prontuários médicos que sofreram alterações na padronização ao longo do tempo, de registro manuscrito, o que trouxe dificuldades para a compreensão. Não foram muitos os dados ausentes dos prontuários devido ao roteiro para o interrogatório sistemático, e possivelmente o valor legal das informações. Uma limitação importante foi a falta de dados sobre a história ocupacional, especialmente para as doenças e acidentes ocupacionais, ou mesmo os riscos do ambiente do trabalho. Assim, deve-se ressaltar que estes dados não traduzem a morbidade ocupacional, mas dados relativos ao estado vital do trabalhador, desde que o registro sistemático de doenças ou disfunções de natureza ocupacional não são realizados nessa etapa da vigilância à saúde do trabalhador.

É escassa a literatura sobre o perfil de morbidade em candidatos a emprego em países industrializados ou em desenvolvimento, embora sejam comuns pesquisas focalizando trabalhadores de empresas em particular. Muitos dos estudos encontrados (Shmunes, 1980; Freeman, 1983; Houghton et al., 1989; Kort \& Dijk, 1997) tratam de discussões sobre o valor dos exames pré-admissionais para a promoção da saúde e/ou prevenção de problemas de saúde entre os trabalhadores. Nos Estados Unidos, em uma avaliação nacional de serviços de vigilância de medicina ocupacional na indústria 
com os dados de exames periódicos, apresentam-se dados que revelam maiores prevalências de problemas de saúde entre as empresas de maior porte e do ramo de manufaturas, em comparação com o da construção civil e as demais empresas, embora prevalências de problemas de saúde específicos não tenham sido apresentadas (Conway et al., 1993). É surpreendente que pesquisadores tenham ignorado a situação de saúde do trabalhador à entrada do emprego, pois esta etapa compreende o componente de seleção do chamado "efeito do trabalhador sadio” (Wilcosky \& Wing, 1987), já apontado como expressão de desigualdades sociais (Koskela, 1982; Santana, 1996). Os principais achados deste estudo sustentam a ne-

\section{Agradecimentos}

Este trabalho não poderia ter sido realizado sem a ajuda da equipe da unidade de saúde, especialmente os funcionários do setor de arquivo médico. Agradecemos também, aos trabalhadores que contribuíram anonimamente com as informações sobre o seu estado de saúde, cujo bem estar é o objetivo último desta pesquisa. cessidade de implementação de programas integralizados de promoção à saúde dos trabalhadores que enfoquem, além dos riscos e enfermidades ocupacionais, a prevenção de doenças infecto-contagiosas e de fatores de risco conhecidos para doenças crônicas. Motoristas parecem pertencer a um grupo em risco especial e devem ser alvo de programas específicos, seja de prevenção, seja em relação às pesquisas voltadas para a identificação de fatores ocupacionais para a obesidade e hipertensão. Além disso, foi possível demonstrar nesse trabalho, que dados de exames admissionais, periódicos e demissionais, podem gerar informações epidemiológicas relevantes para o diagnóstico do estado vital e morbidade de trabalhadores.

\section{Referências}

BELKI'C, K.; SAVI'C, C.; THEORELL, T.; RAKI'C, L.; ERCEGOVAC, D. \& DJORDJEVI'C, M., 1994. Mechanisms of cardiac risk among professional drivers. Scandinavian Journal of Work, Environment and Health, 20:73-86.

BERTOLAMI, M. C.; FALUDI, A. A.; LATORRE, M. R. \& ZAIDAN FILHO, T., 1993. Perfil lipídico de empregados da indústria metalúrgica e suas relações com outros fatores de risco. Arquivos Brasileiros de Cardiologia, 60:293-299.

BRASIL, 1978. Norma Regulamentadora 7, Portaria no 3.214, de 08 de Junho de 1978. Aprova as normas regulamentadoras (NR) do Capítulo V, Título II, da Consolidação das Leis do Trabalho, relativas à Segurança e Medicina do Trabalho. Brasília, DF: Secretaria de Segurança e Saúde no Trabalho, Ministério do Trabalho.

CONWAY, H.; SIMMONS, J. \& TALBERT, T., 1993. The purposes of occupational medical surveillance in US industry and related health findings. Journal of Occupational Medicine, 35:670-686. 
CORDEIRO, R., 1991. Pressão Arterial Diastólica entre Motoristas e Cobradores de Campinas, Usuários de um Serviço de Saúde Ocupacional. Dissertação de Mestrado, Campinas: Faculdade de Ciências Médicas, Universidade de Campinas.

FERREIRA, C. S.; FERREIRA, M. U. \& NOGUEIRA, M. R., 1994. The prevalence of infection by intestinal parasites in an urban slum in São Paulo, Brazil. Journal of Tropical Medicine \& Hygiene, 97:121127.

FREEMAN, C., 1983. Importance of pre-employment physicals. Occupational Health Nursing, 31:35-37.

GOMES, J. R., 1982. Alimentação e trabalho. Revista Brasileira de Saúde Ocupacional, 40:13-15.

GONZALEZ, J. S.; RIVERA, A. E. C.; NORIEGA, J. L. Q.; MORADO, Z.;ANDRADE, M.; CABRERA, M. \& PEREZ, I. J., 1998. Correlación de ejercicio fisico, anemia y celularidad hemática: Un modelo diagnóstico y epidemiológico. Revista Mexicana de Patologia Clínica, 45:17-29.

HOUGHTON, A. M.; EDMONSON-JONES, J. P. \& HARRIS, L. A., 1989. Pre-employment screening: Use or ornament? Journal of Occupational Medicine, 39:51-55.

JOVANOVI'C, J.; BATANJAC, J.; JOVANOVI'C, M.; BULAT, P.; TORBICA, N. \& VESOVI'C, 1998. Occupational profile and cardiac risks: Mechanisms and implications for professional drivers. International Journal of Occupational Medicine and Environmental Health, 11:145-152.

JOVANOVI'C, J.; BATANJAC, J. \& JOVANOVI'C, M., 1999. The influence of cardiovascular diseases of the drivers on the occurrence of traffic accidents. Vojnosanitetski Pregled, 56:3-8.

KLEIN, C. H.; COUTINHO, E. S. F. \& CAMACHO, L. A. B., 1986. Variação da pressão arterial em trabalhadores de uma siderúrgica. Cadernos de Saúde Pública, 2:212-226.

KORT, W. \& DIJK, F., 1997. Preventive effectiveness of pre-employment medical assessments. Occupational and Environmental Medicine, 54:1-6.

KOSKELA, R. S., 1982. Occupational mortality and morbidity in relationto selective turnover. Scandinavian Journal of Work, Environment and Health, 8(Sup. 1):34-39.

LABERGE-NADEAU, C.; DIONNE, G.; MAAG, U.; DESJARDINS, D.; VANASSE, C. \& EKO'E, J. M., 1996. Medical conditions and the severity of commercial motor vehicle drivers' road accidents. Accident Analysis and Prevention, 28:43-51.

LESSA, I., 1998. O Adulto Brasileiro e as Doenças da Modernidade - Epidemiologia das Doenças Crônicas Não-Transmissíveis. São Paulo/Rio de Janeiro: Editora Hucitec/ABRASCO.

MATURU, N. R. \& ILO (International Labour Organization), 1979. Nutrition and labour productivity. International Labour Review, 118:1-12.

MELHADO, J. C.; DINE, J. M.; ROSA, A. A. P.; SANTOS, M. T. R. S. \& VAISMAN, M., 1983. Influência do estado nutricional na produtividade de trabalhadores da construção civil no município do Rio de Janeiro. Revista Brasileira de Saúde Ocupacional, 11:43-49.

MILLER, O., 1991. Hemograma - Série vermelha. In: Laboratório para o Clínico (O. Miller, R. R. Gonçalves, G. F. Pecego, J. F. Penteado \& J. M. R. Alves, org.), pp.76-82, 7ạ Ed., Rio de Janeiro/São Paulo: Livraria Atheneu Editora.

NIH (National Institutes of Health)/NATIONAL HEART, LUNG, AND BLOOD INSTITUTE/NATIONAL HIGH BLOOD PRESSURE EDUCATION PROGRAM, 1997. Blood pressure measurement and clinical evaluation. In: The Sixth Report of the Joint National Committee on Prevention, Detection, Evaluation, and Treatment of High Blood Pressure, pp. 11-18, Bethesda: NIH Publication.

PEARCE, N.; MATOS, E.; KOIVUSAL, M. \& WING, S., 1996. Industrialization and health. In: Occupational Cancer in Developing Countries (N. Pearce, E. Matos, H. Vainio, P. Boffetta \& M. Kogevinas, eds.), International Agency for Research on Cancer (IARC) Scientific Publications no 129, pp. 7 22, Lyon: IARC.

RAGLAND, D. R.; WINKLEBY, M. A.; SCHWALBE, J.; HOLMAN, B. L.; MORSE, L.; SYME, S. L. \& FISHER, J. M., 1987. Prevalence of hypertension in bus drivers. International Journal of Epidemiology, 16:208-214.

SANTANA, V. S., 1996. Globalização e a saúde do trabalhador no Brasil - O que está acontecendo e o que poderá acontecer. In: 4a Reunião da Sociedade Brasileira para o Progresso da Ciência (SBPC), Resumos, pp. 41-46. Feira de Santana: SBPC.

SHMUNES, E., 1980. The importance of pre-employment examination in the prevention and control of occupational skin disease. Journal of Occupational Medicine, 22:407-409.

SINGER, P., 1976. A repartição da renda na AMS. In: Demanda por Alimentos na Área Metropolitana de Salvador (P. Singer, E. M. Oliveira, M. A. Luchesi, J. L. F. Santos \& S. Nigro, org.), pp. 59-61, São Paulo: Centro de Estudos Brasileiros para o Planejamento/Brasiliense.

STOCKES, M. E.; DAVIS, C. S. \& KOCH, G. C., 1995. Categorical Data Analysis Using the SAS System. Cary: SAS Institute.

U. S. DEPARTMENT OF COMMERCE, 1980. Standard Occupational Classification Manual. Washington, D.C.: Office of Federal Statistical Policy and Standards, U. S. Department of Commerce.

WILCOSKY, T. \& WING, S., 1987. The healthy worker effect - Selection of workers and work forces. Scandinavian Journal of Work, Environment and Health, 13:70-72.

WORLD HEALTH ORGANIZATION CONSULTATION ON OBESITY, 1998. Defining the problem of overweight and obesity. In: Obesity: Preventing and Managing the Global Epidemic. Report of a WHO Consultation on Obesity, pp. 7-16, Geneva: WHO. 